\title{
PERFECT PRE-IMAGES OF COLLECTIONWISE NORMAL SPACES
}

\author{
PEG DANIELS
}

\begin{abstract}
We show that the following are true for perfect maps: (1) collectionwise normality with respect to compact (Lindelöf) sets is preserved inversely; (2) collectionwise normality with respect to countably compact $\left(\omega_{1}\right.$ compact) sets is preserved inversely if and only if the domain space is normal with respect to countably compact $\left(\omega_{1}\right.$ - compact) sets; and (3) if $P$ is any property such that (i) $P$ is preserved by perfect maps, (ii) the free union of spaces satisfying $P$ also satisfies $P$, (iii) $P$ is closed hereditary, and (iv) $P$ plus collectionwise normality implies countable metacompactness, then collectionwise normality with respect to closed $P$-sets is preserved inversely if the domain space is normal with respect to closed $P$-sets. Examples of such a property $P$ are paracompactness, submetacompactness, stratifiability and countable metacompactness.
\end{abstract}

1. Introduction. It is well known that collectionwise normality is not preserved inversely by perfect maps $[\mathbf{R}]$. We show that collectionwise normality with respect to compact (Lindelöf) sets is preserved inversely. Also, collectionwise normality with respect to many other properties is preserved inversely if the domain is assumed to be normal with respect to those properties: we show collectionwise normality with respect to countably compact ( $\omega_{1}$-compact) sets is preserved inversely if and only if the domain space is normal with respect to countably compact $\left(\omega_{1^{-}}\right.$compact) sets. Also, if $P$ is any property such that (i) $P$ is preserved by perfect maps, (ii) the free union of spaces satisfying $P$ also satisfies $P$, (iii) $P$ is closed hereditary, and (iv) $P$ plus collectionwise normality implies countable metacompactness, then collectionwise normality with respect to closed $P$-sets is preserved inversely if the domain space is normal with respect to closed $P$-sets. Examples of such a property $P$ are paracompactness, submetacompactness, stratifiability, and countable metacompactness.

Since a closed hereditary property $Q$ is preserved inversely by perfect maps with completely regular domains if and only if $X \times Y$ has property $Q$ whenever $X$ is completely regular with property $Q$ and $Y$ is compact; see $[\mathbf{B}]$, the above results naturally translate into results about special kinds of collectionwise normality in products with a compact factor. Normality, and to some extent collectionwise normality, of products with a compact factor have been studied before, see e.g. [R and $\mathbf{M}$ ], with a typical result being that if $X$ is $\kappa$-paracompact and collectionwise normal, and $Y$ is compact with weight $\leq \kappa$, then $X \times Y$ is collectionwise normal [M] (a space $X$ is $\kappa$-paracompact if and only if every open cover of size $\leq \kappa$ has a locally finite open refinement).

Received by the editors December 10, 1984 and, in revised form, May 2, 1985

1980 Mathematics Subject Classification. Primary 54C10, 54D15. 
We assume all spaces under consideration are Hausdorff. A separation of a collection $\{H(\alpha): \alpha \in \Lambda\}$ is a pairwise disjoint collection $\{U(\alpha): \alpha \in \Lambda\}$ of open sets such that $H(\alpha) \subset U(\alpha)$ for all $\alpha$. A space $X$ is collectionwise Hausdorff (CWH) if every discrete set of points in $X$ has a separation; it is collectionwise normal $(\mathrm{CWN})$ if every discrete collection of closed sets has a separation. Similarly, if $P$ is a property, we say that $X$ is collectionwise normal with respect to $P$-sets $(C W N$ w.r.t. $P$-sets $)$ if every discrete collection consisting of $P$-sets has a separation.

2. Results. We first show that $\mathrm{CWH}$ and $\mathrm{CWN}$ w.r.t. compact (Lindelöf) sets are preserved inversely. First, we have a preliminary lemma:

LEMMA 1. If $f: X \rightarrow Y$ is a perfect map onto $Y$, and $Y$ is regular with respect to Lindelöf sets, then $X$ is normal with respect to Lindelöf sets.

To prove Lemma 1, first show that $X$ is regular with respect to Lindelöf sets, and then mimic the proof that regular Lindelöf spaces are normal.

THEOREM 2. If $f: X \rightarrow Y$ is a perfect map onto $Y$, and $Y$ is $C W H$ (CWN w.r.t. compact sets, $C W N$ w.r.t. Lindelof sets), then so is $X$.

Proof. The CWH case is easy. Suppose $H$ is a discrete set of points in $X$. $f(H)$ is locally finite in $Y$, hence discrete, since $Y$ is Hausdorff, and so may be separated. Pulling back the separation to $X$ gives us a collection $\mathcal{U}$ of pairwise disjoint open sets such that for each $U$ in $U, U \cap H$ is finite. Since $X$ is Hausdorff, the points of each $U \cap H$ can be separated, thus $H$ can be separated.

Now suppose $Y$ is CWN w.r.t. compact (Lindelöf) sets. Suppose $\not H=\{H(\alpha): \alpha \in$ $\Lambda\}$ is a discrete collection of compact (Lindelöf) sets in $Y$.

We form the usual equivalence classes on $\not^{\prime \prime}=\{f(H(\alpha)): \alpha \in \Lambda\}$, a locally finite, hence star-finite (star-countable), collection of compact (Lindelöf) sets in $Y$, by letting $S_{\alpha, 0}^{\prime}=\{f(H(\alpha))\}$, and for each $n \in \omega, S_{\alpha, n+1}^{\prime}=\{f(H(\beta))$ : there exists $\gamma \in \Lambda$ such that $f(H(\gamma)) \in S_{\alpha, n}^{\prime}$ and $\left.f(H(\gamma)) \cap f(H(\beta)) \neq \varnothing\right\}-\bigcup_{m \leq n} S_{\alpha, m}^{\prime}$. Let $S_{\alpha}^{\prime}=\bigcup_{n \in \omega} S_{\alpha, n}^{\prime}$. Note that each $S_{\alpha, n}^{\prime}$ is finite (countable). Clearly the $S_{\alpha}^{\prime}$ 's partition $\mathcal{H}^{\prime}$, so let $\left\{S_{\alpha}^{\prime}: \alpha \in \Gamma\right\}$ enumerate the distinct elements of $\left\{S_{\alpha}^{\prime}: \alpha \in \Lambda\right\}$. For each $\alpha \in \Gamma$, let $S_{\alpha}=\left\{H(\beta): f(H(\beta)) \in S_{\alpha}^{\prime}\right\}, S_{\alpha, n}=\left\{H(\beta): f(H(\beta)) \in S_{\alpha, n}^{\prime}\right\}$, and $S_{\alpha, n}=\bigcup S_{\alpha, n}^{\prime}$.

Partition $\mathcal{H}$ into countably many pieces by letting $\mathcal{H}(n)=\bigcup_{\alpha \in \Gamma} S_{\alpha, n}$. We first show that the $H(n)$ 's can be separated, i.e., for each $n \in \omega$ there is an open set $U(n)$ in $X$ such that $\bigcup \forall(n) \subset U(n)$ and $\{U(n): n \in \omega\}$ is pairwise disjoint. It suffices to show that for each $n, \mathcal{H}(n)$ can be separated from $\bigcup\{\bigcup \mathscr{H}(m): m>n\}$. We do this in three steps. First, the fact that $\left\{S_{\alpha, n+2 j}: \alpha \in \Gamma\right.$ and $\left.j \in \omega\right\}$ is a discrete collection of compact (Lindelöf) sets enables us to separate $\bigcup\left\{S_{\alpha, n}: \alpha \in \Gamma\right\}$ from $\bigcup\left\{S_{\alpha, n+2 j}: \alpha \in \Gamma\right.$ and $\left.j \geq 1\right\}$ in $Y$, and hence we can separate $\bigcup \mathcal{H}(n)$ from $\bigcup\{\bigcup \mathcal{H}(n+2 j): j \geq 1\}$ in $X$. Also note this shows that $\left\{\bigcup S_{\alpha, n}: \alpha \in \Gamma\right\}$ can be separated. Next, for a similar reason, $\bigcup H(n)$ can be separated from $\bigcup\{\bigcup \mathcal{H}(n+2 j+1): j \geq 1\}$. Finally, to see that $\bigcup \mathcal{H}(n)$ can be separated from $\bigcup \mathcal{H}(n+1)$, first note that $\left\{S_{\alpha, n} \cup S_{\alpha, n+1}: \alpha \in \Gamma\right\}$ is a discrete collection of compact (Lindelöf) sets, and so can be separated. This gives back in $X$ a separation of $\left\{\bigcup S_{\alpha, n} \cup \bigcup S_{\alpha, n+1}: \alpha \in \Gamma\right\}$. Since each $S_{\alpha, n} \cup S_{\alpha, n+1}$ is a finite (countable) collection of compact (Lindelöf) sets, these sets can be separated from each other since $X$ is Hausdorff (normal with respect to Lindelöf sets, and hence CWN with respect 
to countable discrete collections of Lindelöf sets). Putting these three separations together enables us to separate $\bigcup \mathcal{H}(n)$ from $\bigcup\{\mathcal{H}(m): m>n\}$. Thus the $\mathcal{H}(n)$ 's can be separated from each other.

We also noted that each $\left\{S_{\alpha, n}: \alpha \in \Gamma\right\}$ can be separated in $Y$. This gives a separation in $X$ of $\left\{\bigcup S_{\alpha, n}: \alpha \in \Gamma\right\}$. Since each $S_{\alpha, n}$ can be separated, the elements of each $\forall(n)$ can be separated from each other. Thus $\nLeftarrow$ can be separated in $X$.

For Theorem 2 we needed to be able to separate two points, or two compact sets, or two Lindelöf sets. The ability to do so was an automatic byproduct of the assumptions on $Y$ and the perfectness of the map. This is not always the case. In Theorem 3 we need to assume additional normality-like properties on the domain space in order to get the CWN under consideration preserved inversely.

THEOREM 3. If $f: X \rightarrow Y$ is a perfect map onto $Y, Y$ is $C W N$ w.r.t. countably compact $\left(\omega_{1}\right.$-compact) sets, and $X$ is normal w.r.t. countably compact $\left(\omega_{1}\right.$-compact) sets, then $X$ is $C W N$ w.r.t. countably compact ( $\omega_{1}$-compact) sets.

It is easy to check that if $f: X \rightarrow Y$ is perfect and $\{H(\alpha): \alpha \in \Lambda\}$ is a discrete collection of countably compact $\left(\omega_{1}\right.$-compact) sets in $X$, then $\{f(H(\alpha)): \alpha \in \Lambda\}$ is a locally finite collection of countably compact ( $\omega_{1}$ - compact) sets, and hence is a star-finite (star-countable) collection. Thus the proof for countably compact sets is parallel to the proof for compact sets, and the proof for $\omega_{1}$-compact sets is parallel to the proof for Lindelöf sets. The only difference is that in the third step of separation, to be able to separate a finite (countable) number of countably compact $\left(\omega_{1}\right.$-compact) sets, we need to assume that $X$ is normal with respect to countably compact ( $\omega_{1}$-compact) sets. There is no analogue of Lemma 1 for these cases, as the following example shows.

EXAMPLE 4. The projection map $\pi: \omega_{1} \times\left(\omega_{1}+1\right) \rightarrow \omega_{1}$ is a perfect map onto a CWN space. However, $\omega_{1} \times\left\{\omega_{1}\right\}$ and $\left\{(\alpha, \alpha): \alpha \in \omega_{1}\right\}$ are two disjoint closed countably compact sets (since they are each homeomorphic to $\omega_{1}$ ) that cannot be separated.

The above example is typical of examples showing that $\mathrm{CWN}$ is not preserved inversely by perfect maps. In the light of Theorem 3 , this brings up the following question:

QUESTION 5. Is there a normal space $X$ and a perfect image of $X$ such that the image is CWN, but $X$ is not?

In order to comment on this question further, and lead into our next theorem, we need some background definitions and results, found in [K and SK].

DEFINITION 6. A space is expandable (discretely expandable) if for every locally finite (discrete) collection $\{F(\alpha): \alpha \in \Lambda\}$ of subsets of $X$, there exists a locally finite collection $\{G(\alpha): \alpha \in \Lambda\}$ of open subsets of $X$ such that $F(\alpha) \subset G(\alpha)$. If the collection $\{G(\alpha): \alpha \in \Lambda\}$ is only point finite, then the word "almost" precedes the above terminology.

THEOREM [SK]. A space $X$ is expandable if and only if $X$ is discretely expandable and countably paracompact.

THEOREM [SK]. A space $X$ is almost expandable if and only if $X$ is almost discretely expandable and countably metacompact.

THEOREM [SK]. A normal space is $C W N$ if and only if $X$ is discretely expandable. 
Returning to Question 5, it is clear that $X$, and hence its image $Y$, cannot be countably paracompact, since if $Y$ is countably paracompact, it is expandable. It is easy to check that this implies that $X$ is discretely expandable, and hence, since normal, it is also CWN. So to answer Question 5 in the affirmative, we need a perfect map from a non-CWN Dowker space onto a CWN Dowker space. Furthermore, no such example is of the form $\pi: X \times Y \rightarrow X$, for compact $Y$, since Rudin $[\mathbf{R}]$ and Dowker [D] have shown that if $Y$ is infinite and compact, and $X \times Y$ is normal, then $X$ is countably paracompact.

It follows from these background theorems that if a space $X$ is $\mathrm{CWN}$, then it is almost expandable if and only if $X$ is countably metacompact (if and only if $X$ is countably paracompact, by Dowker's theorem). Our first version of Theorem 7 was that CWN w.r.t. closed paracompact sets is preserved inversely assuming a normality-like property on the domain space, but in examining the proof more closely, we realized paracompactness could be generalized to many properties which when paired with CWN give almost expandability. Thus the general form of the theorem is as follows:

THEOREM 7. Suppose $P$ is a property such that

(i) $P$ is preserved by perfect maps,

(ii) a free union of spaces satisfying $P$ also satisfies $P$,

(iii) $P$ is closed hereditary, and

(iv) $P$ plus $C W N$ implies almost expandability.

Then if $f: X \rightarrow Y$ is a perfect map onto $Y, X$ is normal w.r.t. closed $P$-sets, and $Y$ is $C W N$ w.r.t. closed $P$-sets, then $X$ is $C W N$ w.r.t. closed $P$-sets.

For the proof of Theorem 7 we need the following well-known lemma:

LEMMA. If $H=\{H(\alpha): \alpha \in \Lambda\}$ is a locally finite collection of closed P-sets, where $P$ is a property satisfying (i) and (ii) of Theorem 7, then $\bigcup\{H(\alpha): \alpha \in \Lambda\}$ is a P-set.

This lemma follows from the fact that the natural map

$$
f: \bigoplus_{\alpha \in \Lambda} H(\alpha) \rightarrow \bigcup\{H(\alpha): \alpha \in \Lambda\}
$$

is perfect for such a collection $\nVdash$. (See $[\mathbf{E}]$.)

ProOF OF THEOREM 7 . Let $X, Y, f$ be as in the hypothesis, and $P$ a property satisfying (i)-(iv). Suppose $\psi=\{H(\alpha): \alpha \in \Lambda\}$ is a discrete collection of closed $P$-sets. It is easy to check that if we could find for each $\alpha$ a countable collection of open sets in $X,\{U(\alpha, n): n \in \omega\}$, such that $H(\alpha) \subset \bigcup\{U(\alpha, n): n \in \omega\}$ and $H(\alpha) \cap \bigcup\{U(\beta, n): \beta \neq \alpha\}=\varnothing$, then

$$
\{\bigcup\{(U(\alpha, n)-\overline{\bigcup\{U(\beta, m): \beta \neq \alpha, m \leq n\}}): n \in \omega\}: \alpha \in \Lambda\}
$$

is a separation of $\psi$.

Since $\mathscr{H}^{\prime}=\{f(H(\alpha)): \alpha \in \Lambda\}$ is a locally finite collection of closed $P$-sets, by the lemma $\bigcup \nvdash^{\prime}$ is a $P$-set. Since $\bigcup \nvdash^{\prime}$ is closed in $Y$, it is CWN w.r.t. closed $P$-sets, and thus CWN, since every closed subset of $U \mathscr{H}^{\prime}$ is a $P$-set by property (iii). By property (iv), let $\{G(\alpha): \alpha \in \Lambda\}$ be a point finite open-in- $\bigcup \nvdash^{\prime}$ expansion of $\mathfrak{H}^{\prime}$. 
For each $\alpha \in \Lambda$, let $J(\alpha)=G(\alpha)-\bigcup\{G(\beta): \beta \neq \alpha\}$. Then $J(1)=\{J(\alpha): \alpha \in \Lambda\}$ is a discrete-in- $\bigcup H^{\prime}$ collection of closed-in- $U \mathcal{H}^{\prime}$ sets, and thus a discrete collection of closed $P$-sets in $Y$. So let $\mathcal{V}(1)=\{V(\alpha): \alpha \in \Lambda\}$ be an open-in- $Y$ separation of $J(1)$. Furthermore, if $p \in J(\alpha)$, then $f^{-1}(p)$ meets only $H(\alpha)$, so since $f$ is closed, we may assume that $V(\alpha)$ meets only $f(H(\alpha))$.

For each $\alpha \neq \beta$ in $\Lambda$, let

$$
J(\alpha, \beta)=((G(\alpha) \cap G(\beta))-\bigcup\{G(\gamma): \gamma \neq \alpha, \beta\})-\bigcup \mathcal{V}(1) .
$$

Then $J(2)=\{J(\alpha, \beta): \alpha \neq \beta$ in $\Lambda\}$ is a discrete collection of closed $P$-sets in $Y$, so let $\mathcal{V}(2)=\{V(\alpha, \beta): \alpha \neq \beta$ in $\Lambda\}$ be an open-in- $Y$ separation of $J(2)$. Since $p \in J(\alpha, \beta)$ implies that $f^{-1}(p)$ meets at most $H(\alpha)$ and $H(\beta)$, we may assume that $V(\alpha, \beta)$ meets at most $f(H(\alpha))$ and $f(H(\beta))$.

Continue in this way for countably many steps until $\bigcup H^{\prime}$ has been exhausted.

We show next that we may assume without loss of generality that each $\mathcal{V}(n)$ is discrete with respect to $\bigcup \mathcal{H}^{\prime}$, i.e., if $p \in \bigcup \mathcal{H}^{\prime}$, then there is an open set in $Y$ containing $p$ that meets at most one element of the collection. Since $\bigcup J(n)$ and $(\overline{\bigcup \mathcal{V}(n)}-\bigcup \mathcal{V}(n)) \cap\left(\bigcup \mathcal{H}^{\prime}\right)$ are disjoint closed $P$-sets, let $W$ be open in $Y$ such that $W$ contains the first set and its closure misses the second set. It is easy to show that

$$
\left\{V(\alpha(1), \ldots, \alpha(n)) \cap W:\{\alpha(1), \ldots, \alpha(n)\} \in[\Lambda]^{n}\right\}
$$

is a separation of $J(n)$ that is discrete with respect to $\bigcup \mathscr{H}^{\prime}$. So we assume that $\mathcal{V}(n)$ itself has this property.

We have assumed that each $f^{-1}(V(\alpha(1), \ldots, \alpha(n)))$ meets at most $H(\alpha(1)), \ldots$, $H(\alpha(n))$. By the same type of argument as that in the paragraph above, we may assume without loss of generality that $f^{-1}(\overline{V(\alpha(1), \ldots, \alpha(n))})$ meets at most $H(\alpha(1)), \ldots, H(\alpha(n))$.

Since $X$ is normal with respect to closed $P$-sets, let $U(\alpha(1), \ldots, \alpha(n) ; i)$ be an open set in $X$ containing $H(\alpha(i))$ such that $\{U(\alpha(1), \ldots, \alpha(n) ; i): i \leq n\}$ is pairwise disjoint.

We claim that if $\alpha \in \Lambda$ and $p \in H(\alpha)$, there is an open set $V$ containing $p$ such that if $\{\alpha(1), \ldots, \alpha(n)\} \in[\Lambda]^{n}$ and $i \leq n$ is such that

$$
V \cap f^{-1}(V(\alpha(1), \ldots, \alpha(n))) \cap U(\alpha(1), \ldots, \alpha(n) ; i) \neq \varnothing,
$$

then $\alpha=\alpha(i)$; this gives us that $H(\alpha) \cap \overline{\bigcup\{U(\beta, n): \beta \neq \alpha\}}=\varnothing$, where

$$
\begin{aligned}
& U(\beta, n)=\bigcup\left\{f^{-1}(V(\alpha(1), \ldots, \alpha(n))) \cap U(\alpha(1), \ldots, \alpha(n) ; i):\right. \\
& \left.\{\alpha(1), \ldots, \alpha(n)\} \in[\Lambda]^{n}, i \leq n, \text { and } \beta=\alpha(i)\right\} .
\end{aligned}
$$

It is easy to check that $H(\alpha) \subset \bigcup\{U(\alpha, n): n \in \omega\}$, and so

$$
\left\{\bigcup_{n \in \omega}(U(\alpha, n)-\overline{\bigcup\{U(\beta, m): \beta \neq \alpha, m \leq n\}}): \alpha \in \Lambda\right\}
$$

is a separation of $\mathcal{H}$. To show the claim, suppose $\alpha \in \Lambda$ and $p \in H(\alpha)$. Since $\mathcal{V}(n)$ is discrete with respect to $U \mathcal{H}^{\prime}$, we may let $U$ be an open set containing $p$ that meets only the unique element, if any, of $\left\{f^{-1}(V(\alpha(1), \ldots, \alpha(n))):\{\alpha(1), \ldots, \alpha(n)\} \in\right.$ 
$\left.[\Lambda]^{n}\right\}$ of which $p$ is in the closure. If there is no such element, let $V=U$. If there is such an element, let $f^{-1}(V(\alpha(1), \ldots, \alpha(n)))$ be the one. Since

$$
p \in \overline{f^{-1}(V(\alpha(1), \ldots, \alpha(n)))} \subset f^{-1}(\overline{V(\alpha(1), \ldots, \alpha(n))}),
$$

we have that $\alpha=\alpha(i)$ for some $i \leq n$, and so $p \in U(\alpha(1), \ldots, \alpha(n) ; i)$. Then $V=U \cap U(\alpha(1), \ldots, \alpha(n) ; i)$ has the desired properties.

Thus $\nvdash$ can be separated, and so $X$ is CWN w.r.t. closed $P$-sets.

In the light of the remarks before Theorem 7 , obvious examples of such a property $P$ are properties satisfying (i)-(iii) which either imply countable metacompactness, e.g. (countable) paracompactness, (countable) metacompactness, metrizability, and stratifiability, or which imply countable metacompactness when CWN is also assumed, e.g. (countable) subparacompactness, (countable) submetacompactness, developability, semistratifiability, and semimetrizability.

In the proof of Theorem 7, the fact that $X$ is normal with respect to closed sets is used quite strongly. Example 4 shows that at least in some cases this is necessary, e.g. if $P$ is countable paracompactness, but we do not know if this always the case. A typical question along these lines is the following:

QUESTION 8. Is there an example of a space that is not normal with respect to closed paracompact sets, yet has a perfect image that is CWN w.r.t. closed paracompact sets?

In a typical method of producing counterexamples, one takes the product of a compact space and a space $X$ with the desired property, and shows that there are two disjoint closed sets, at least one of which is homeomorphic to $X$, that cannot be separated. (Note: the projection onto $X$ is perfect.) But of course in this case, if the homeomorph to $X$ is paracompact, then so is the whole product. Since a closed hereditary property $P$ is inversely preserved by perfect maps with completely regular domains if and only if $X \times Y$ has property $P$ whenever $X$ is completely regular with property $P$ and $Y$ is compact, a natural place to look for a counterexample is at this type of product.

In terms of perfect maps preserving various types of CWN directly, it is easy to see that CWN w.r.t. $P$-sets is preserved, where $P$ is any property preserved inversely by perfect maps, e.g. compactness, Lindelöfness, paracompactness, countable compactness, countable metacompactness, etc. CWH is clearly not preserved: take any $\mathrm{CWH}$ space in which there is a discrete collection of compact sets that cannot be separated [W] and identify the compact sets to points. CWN w.r.t. metrizable sets is also not preserved, as $\mathrm{S}$. Watson $[\mathbf{W}]$ showed in an example of a normal space that is CWN w.r.t. metrizable sets, but has a discrete collection of compact sets that cannot be separated; identify the compact sets to points to get a counterexample.

\section{REFERENCES}

[B] D. K. Burke, Closed mappings, Surveys in General Topology, Academic Press, New York, 1980, pp. 1-32.

[D] C. H. Dowker, On countably paracompact spaces, Canad. J. Math. 3 (1951), 219-224.

[E] R. Engelking, General topology, Polish Scientific Publishers, Warsaw, 1977.

[K] L. Krajewski, Expanding locally finite collections, Canad. J. Math. 23 (1971), 58-68.

[M] K. Morita, Paracompactness and product spaces, Fund. Math. 53 (1961), 223-236. 
[R] M. E. Rudin, Products with a compact factor, Topology Appl. 5 (1975), 45-59.

[SK] J. C. Smith and L. Krajewski, Expandability and collectionwise normality, Trans. Amer. Math. Soc. 160 (1971), 437-451.

[W] S. Watson, Separation and coding, preprint.

Department of Mathematics, University of Toronto, Toronto, Ontario M5S 1A1, CANADA

Current address: Department of Mathematics, Auburn University, Auburn, Alabama 36849 